\title{
Die Beschäftigung mit den spanischen Humanisten im Deutschland des 19. Jahrhunderts
}

Strosetzki, Christoph

First published in:

Das Spanieninteresse im deutschen Sprachraum, S. 22 - 33, Vervuert Verlag, Vervuert 1989 ISBN 3-89354-827-0

Münstersches Informations- und Archivsystem multimedialer Inhalte (MIAMI) URN: urn:nbn:de:hbz:6-72439618597 


\title{
Die Beschäftigung mit den spanischen Humanisten im Deutschland des 19. Jahrhunderts
}

\author{
Christoph Strosetzki \\ Düsseldorf
}

Die Renaissance ist in der Hispanistik früh vernachlässigt worden. Dies im einzelnen nachzuvollziehen wäre wenig ergiebig. Daher soll im folgenden der geistige Horizont skizziert werden, der für jene wissenschaftsgeschichtliche Entwicklung verantwortlich war, die eine angemessene Würdigung der Humanisten verhindert hat. Es wird dabei weniger von der Beurteilung einzelner Humanisten die Rede sein. Vielmehr soll gezeigt werden, welche Konsequenzen die Betonung des Volkstümlichen in der deutschen Romantik auf die Interessengewichtung der Hispanistik hatte. Da sich die gelehrte Literatur des spanischen Humanismus mit der griechischen und römischen Vergangenheit befaßt, und als Kapitel der Geschichte der Altphilologie erscheint, ist auch das Konkurrenzverhältnis zwischen Alt- und Neuphilologie einzubeziehen. Auch soll gezeigt werden, wie sich die Bevorzugung bestimmter literarischer Formen auf die Beurteilung der humanistischen Literatur auswirkte. Abschließend wird die Bewertung des Einflusses der Kirche auf die Entfaltung des Humanismus vorgeführt.

\section{Klemperer und seine These}

Eine Renaissance gäbe es in Spanien nicht, hatte noch 1927 V. Klemperer behauptet. Er konnte sich dabei auf Morf berufen, der 1909 Iberien als Vorkämpfer eines ungebrochenen Mittelalters sah ${ }^{1}$. Spanien habe zwar Bildungselemente aus der italienischen Renaissance übernommen, jedoch nicht den Bruch mit dem Mittelalter vollzogen. Klemperer ergänzt, Spanien habe durch die Auseinandersetzung mit den Arabern ein außereuropäisches Sonderschicksal erlitten und könne nicht mit Staaten wie Italien verglichen werden, das sich nach einer mittelalterlichen Phase der Konsolidation unter der Herrschaft der Kirche patriotisch auf seine eigene antike Vergangenheit zurückbesinnen konnte. Spanien sei als europäisch-christliches Land erst 1492 geboren - zu einem Zeitpunkt also, als Italien bereits eine Wiedergeburt erleben konnte. Spaniens patriotische Rückbesinnung gelte daher der Reconquista. Ihr Resultat sei nicht die Beschäftigung mit der Antike, sondern eine neue Blüte der Romanzenliteratur gewesen ${ }^{2}$.

Klemperer, der seine These literaturgeschichtlich zu belegen sucht, übersieht, wie sehr er wissenschaftsgeschichtlich der deutschen Romantik verpflichtet ist. Dies ist umso bedeutsamer, als seine These für die Ausklammerung des Humanismus in der. deutschen Hispanistik des frühen 20. Jahrhunderts in ähnlicher Weise verantwortlich zu sein scheint, wie die Romantik für die Ausklammerung dieses Gebietes in der

1 Heinrich Morf, Die Kultur der Gegenwart, Leipzig 1909, Teil 1, Abteil XI, I, S. 220; Victor Klemperer, "Gibt es eine spanische Renaissance?" in: Logos 16, 2, 1927, S. 129-161

2 Ebda., S. 142 ff 
deutschen Hispanistik des 19. Jahrhunderts ${ }^{3}$. Es sei daher im folgenden kurz auf den Romantiker Friedrich Schlegel und auf Johann Gottfried von Herder als den Wegbereiter der Romantik eingegangen.

\section{Von Herder zur Romantik Schlegels}

Herder, der seit 1777 bei Joachim Bertuch Spanisch lernte, hatte die iberische Welt in Weimar entdeckt, als er seine Volksliedsammlung bearbeitete und Gleim um die Übermittlung spanischer Romanzen bat. Ihnen widmete er besonders ausführlichen Raum, da er sie für das Zeugnis eines ursprünglichen Volkscharakters hielt, bei dem Dichtung, Volk und Klima übereinstimmten ${ }^{4}$.

Mit den maurischen Romanzen traten auch die Araber ins Blickfeld. Herder sieht sie als Lehrer Europas und den mittelalterlichen Ritterkult im wesentlichen durch arabischen Geist bestimmt ${ }^{5}$. Tapferkeit, Freiheitsliebe, Ehrgefühl und vergeistigte Religiosität seien prägend gewesen für den Nationalgeist der Araber. In ihm sah Herder 1777 in seiner Schrift über die Wirkung der Dichtung auf die Sitten der Völker neben christlicher Religion und nordischem Geschmack die dritte Komponente des mittelalterlichen Geistes. Poesie sei bei den Arabern - wie später im ritterlichen Leben - nicht isoliertes Kulturgebiet, sondern Bestandteil des alltäglichen Lebens.

Wie Herder betrachtet der Romantiker Friedrich Schlegel in seiner frühen Phase Literatur in erster Linie als Zeugnis des Volkscharakters. Seine Beispiele bezieht er jedoch nicht aus der Romanzentradition, sondern zunächst aus Cervantes und dann aus Calderón. Auch er betont die Bedeutung der Araber, die Spanien eine eigene Entwicklung ermöglicht haben, sieht jedoch im Leben und in den Tugenden des Mittelalters in erster Linie den Einfluß der Goten.

Im Sinne der Identitätsphilosophie nimmt er eine ursprüngliche Einheit an, die durch den Beginn einer Trennung zerstört wird, die im zerstückelten Europa eine geographische und geschichtliche Dimension erhält. In den Vorlesungen zur Universalgeschichte interpretiert er daher die spanische Geschichte als Entwicklungsgeschichte der germanischen Verfassung in Spanien: Zunächst habe die germanische Verfassung den Geist des Römertums besiegt, sich trotz des arabischen Einflusses behauptet und

3 Nicht nur die spanische Renaissance, sondern die gesamte spanische Literatur wird von Klemperer nicht sehr positiv beurteilt. Im Anschluß an den "Deutsch-spanischen Tag" gibt er zu bedenken, daß Frankreich bis ins 14. Jahrhundert die Führerin der Romania war und dann die Führung an Italien übergab, bis es ab Rabelais wieder den ersten Platz einnahm und nie wieder aufgab. Italien habe nur einmal die Führung des romanischen Geistes besessen, Spanien nie. Es ergebe sich also eine Rangordnung unter den romanischen Literaturen: "Die französiche steht an erster Stelle, an zweiter die italienische und die spanische an dritter." Victor Klemperer, "Die Weltștellung der spanischen Sprache und Literatur" in: Bücherei und Bildungspflege 2, H. 5/6, 1922, S. 188-122, hier S. 121

4 Herders Interesse für die Volkspoesie war der Ausgangspunkt für zahlreiche wissenschaftliche Arbeiten in Deutschland. Vgl. dazu: A. E. (Esser?), "Literarische Wechselwirkungen Spaniens und Deutschlands" in: Deutsche Vierteljahrs-Schrift 1857, 2, S. 86-121, hier S.97

${ }_{5} \mathrm{Zu}$ Herder vgl. Wolfgang Kayser, Die iberische Welt im Denken J.G. Herders, Hamburg 1945 , hier S.33 
christliche Wertvorstellungen erarbeitet. Es folge das Mittelalter, in dem die Verfassung zur Reife kommt. Auch im siglo de oro scheint es noch präsent zu sein. Denn erst im 18. Jahrhundert sei der römische Despotismus mit der französischen Hegemonie zurückgekehrt und habe den Geist der alten Verfassung aufgelöst. Da diese drei Epochen nichts anderes als die Geschichte der germanischen, freiheitlichen Ständeverfassung in Spanien bilden, ist es nicht verwunderlich, daß die Suche nach dem zerstreuten Eigenen auch die Aufnahme und die kritische Selektion der spanischen Literatur leitet ${ }^{6}$.

Wie bei Herder, verleihen also auch bei Schlegel die Auseinandersetzungen mit den Arabern der spanischen Geschichte einen besonderen Charakter. Dadurch habe Spanien die größten Rittertugenden entwickeln können, die sich dort auch durch die entfernte Lage reiner und anhaltender als anderswo in Europa darstellten ${ }^{\top}$ - eine These, die Klemperer später wiederholen sollte.

Das Typische des Mittelalters ist nach Schlegel der romantische Geist. Phantasie habe nicht nur in der Dichtung, sonden auch im wirklichen Leben gelegen - eine Tatsache, aus der Schlegel den Vorzug des Mittelalters gegenüber dem klassischen Altertum mit seinem bloßen Ästhetizismus und Scheinglanz der Formen ableitet ${ }^{8}$. Wenn auch später Schlegel die Phantasie und damit das Mittelalter zugunsten einer eschatologischen Geschichtsauffassung, die nur noch die mystischen Autoren akzeptieren konnten, entwertet, so erschienen ihm zunächst Cervantes und später Calderón als die wichtigsten Vertreter der spanischen Literatur. Die Poesie des Cervantes konnte er als Naturpoesie mit der griechischen vergleichen, die er mit demselben $\mathrm{Be}$ griffsapparat charakterisiert hatte wie jene des Cervantes ${ }^{9}$. Das Werk Calderóns gilt ihm 1811 als Verkörperung der Romantik schlechthin, in dem sich nicht nur Christliches und Phantasie, sondern auch Verankerung im geschichtlichen Boden, nationaler Charakter wie naturerwachsener Ausdruck vereinten ${ }^{10}$.

Man kann sich fragen, wie es zum Interesse der Romantiker an Spanien kam. Sicherlich spielt eine Rolle, daß sich auch die Philosophen des deutschen Idealismus gern auf Spanien beriefen, und Schelling, dessen System des transzendentalen Idealismus vom Hispanisten Bouterwek rezensiert wurde, Calderón als Sophokles der geteilten Welt bezeichnet, oder. Hegel in seiner Ästhetik den Cid immer wieder mit

${ }^{6}$ Zu Schlegel vgl.: Ricardo Blanco Unzué, Die Aufnahme der spanischen Literatur bei Friedrich Schlegel, Frankfurt a.M., Bern 1981, hier S. 172; im Gegensatz zu Bouterwek, der mit seinem Ausspruch "somos hermanos" einer künfligen Ergänzung von deutschem Gemüt und spanischer Phantasie Ausdruck verleiht, sucht Schlegel das Echte und das südliche Feuer in einer Vergangenheit. Vgl.: Ebda., S. 169

7 Vgl. ebda., S. $180 \mathrm{ff}$

8 Vgl. ebda., S. $188 \mathrm{ff}$

9 Vgl. ebda., S. 333

10 Vgl. ebda., S. 490; schon 1857 wird den Romantikern vorgeworfen, zu Unrecht das Drama Calderóns als spanisch-national bezeichnet zu haben. Es sei "vielmehr ein Erzeugnis der Verbildung der höheren Stände" A. E., Literarische Wechselwirkungen Spaniens und Deutschlands, a.a.O., S. 99 
der Mias vergleicht ${ }^{11}$. Aber auch die politische Situation mag eine Rolle gespielt haben. Spanien und Deutschland waren im Kampf gegen Napoleon geeint. Spanien und England erschienen als Vorbild für die deutsche Befreiung. Nicht zuletzt darauf mag es zurückzuführen sein, daß man in Deutschland immer mehr englische Reiseberichte von Spanien rezipierte, die zumeist ein positiveres Bild zeigten, als jene französischen von Saint-Simon, Montesquieu und Voltaire, die die "Leyenda negra" wiederbelebt hatten. Auch zur Tatsache, daß die Franzosen der Aufklärung die emanzipatorischen Ansätze der Humanisten des 16. Jahrhunders aufgriffen, konnte man in Opposition treten, wenn man das christliche Mittelalter und seine Ausgestaltung in Spanien thematisierte, wie es die deutschen Romantiker taten.

\section{Volkstypisches}

Die schon bei Herder und Schlegel beobachtete Betonung der Volkstümlichkeit findet sich auch in der Mitte des Jahrhunderts in den Anmerkungen von Ferdinand Wolf zur spanischen Übersetzung von Bouterweks, Literaturgeschichte. In seiner Gliederung der spanischen Nationalliteratur unterscheidet er zwei Hauptperioden: die vor und die nach dem Beginn des 16. Jahrhunderts. Die erste Periode lasse sich als Mittelalter bezeichnen und sei geprägt durch die "spontane Volkspoesie" der Romanzen ${ }^{12}$. In der zweiten Periode sei es die erneut auflebende Beschäftigung mit den Volksromanzen, die "die glänzende und reichste Schöpfung der Kunstpoesie dieser Perioden der spanischen Literatur überhaupt: das Nationaldrama" ${ }^{13}$ entstehen ließ.

Die dramatische Literatur, sei es bei Lope oder Calderón, sei in naher Verbindung mit der Volkspoesie geblieben, habe sich deren Formen bedient und war selbst volkstümlich. Diese altnationale Betonung habe dafür gesorgt, daß die italienischen und antiken Einflüsse auf die Lyrik und die "Nachahmung der humanistisch geschulten italienischen Kunstlyrik" ${ }^{14}$ beschränkt blieben. Auch Graf Schack zeigt 1845/46 in seiner Geschichte des spanischen Dramas, daß das wahrhaft Große in der Poesie nur auf dem Boden der Volkstümlichkeit gedeihen kann. Schon damit wendet er sich von den Humanisten der Renaissance, deren Kult des Weisen und Gelehrten, und vom elitären, vom Volk entfernten "philosophe" der französischen Aufklärung ab.

Mit dem Gegensatz zwischen gelehrter und Volksliteratur will auch die Geschichte der spanischen Literatur von G. Baist in Gröbers Grundriß erklären, warum in Spanien Humanismus und Renaissance nicht Fuß fassen konnten. Die Dominanz der Kirche habe dafür gesorgt, daß es keine "geistige Ablösung der Renaissance vom Mittelalter" gegeben habe, und die neuen Anregungen aus Italien aufgenommen wor-

$11 \mathrm{Vgl}$. Werner Brüggemann, Die Spanienberichte des 18. und 19. Jahrhunderts und ihre Bedeutung für die Formung und Wandlung des deutschen Spanienbildes, Münster 1956 (Spanische Forschungen der Görresgesellschaft, 1, 12) S. 77, 129

12 Ferdinand Woif, Studien zur Geschichte der spanischen und portugiesischen Nationalliteratur, Berlin 1859, S. 9

13 Ebda., S. 15

14 Ebda., S. 17 
den seien, "ohne die ältere Tradition preiszugeben"15. Die Spanier jedoch, die als gelehrte Humanisten eigene Wege suchten, wie Vives, Valdés oder Servet, haben sich ins Ausland zurückziehen müssen und dachten ausländisch. Während Spanien in der Gelehrtenrepublik immer nur eine untergeordnete Stellung eingenommen habe, fanden die "Ideale der Bevölkerung" ihren adäquaten Ausdruck zunächst in der Romanze und seien dann vom Drama übernommen worden.

Die beginnende hispanistische Literaturbetrachtung orientiert sich also an der romantischen Suche nach dem Volkstypischen und kann daher nicht die gelehrten Humanisten der Renaissance einbeziehen. Hinzu kommt, daß die humanistischen Autoren nach Italien blicken und die italienische nationale Vergangenheit wieder aufleben lassen, damit also etwas erneuern, das für die spanische Nation untypisch ist.

\section{Neuphilologie - Altphilologie}

Die Beschäftigung mit antiken Autoren gilt als Aufgabe der Altphilologie. Daher ist auch die humanistische Auseinandersetzung mit der Antike nur schwerlich als Gegenstand der sich gerade etablierenden Neuphilologien denkbar. Sie mußte als Kapitel der Geschichte der Altphilologie gesehen werden und war kaum einer Neuphilologie zuzuordnen, die noch Schwierigkeiten hatte, sich gegenüber der traditionsreichen Altphilologie $2 \mathrm{u}$ behaupten. Dies wird umso deutlicher, wenn man bedenkt, daß noch in Gröbers Grundriß die Wissenschaftsgeschichte als eine der Hilfsdisziplinen der Philologie betrachtet wird.

Bereits Friedemann schreibt im 2. Jahrgang des Archiv für das Studium der neueren Sprachen und Literaturen 1847 einen Artikel mit dem Titel "Der modernen Philologie wird fortdauernde Rücksicht auf die antike empfohlen". Die Gemeinsamkeiten zwischen beiden Philologien sieht er nicht zuletzt darin, daß sich Gelehrte wie C. Lachmann und M. Haupt mit beiden gleichzeitig beschäftigen ${ }^{16}$. Die Altphilologie wünscht er als notwendige Ergänzung der Neuphilologie. Er zitiert Mager, der als Vorkämpfer der modernen Philologie der klassischen den Vorrang läßt und in ihr das schönste Erbteil verkörpert sieht. Es "sei keinem", fordert er, "der sich Philologe nennen will, der Zugang zu einem anderen Volke gestattet, er habe denn seinen Weg dahin über Rom und Athen genommen."17

Das klassische Altertum sei lange Zeit zugleich Quelle von Bildung und moralischer Erziehung gewesen. Seine Lehrer hatten als Kirchendiener zunächst dem theologischen Stand angehört. Daher soll sich auch die moderne Philologie zunächst an solche Nationen halten, "bei denen ein Schöner und Guter mit Nutzen für eigene Geistesund Gemüthsbildung Wohnung aufschlagen kann" ${ }^{18}$. Damit wird der pädagogische

15 Gottfried Baist, "Die spanische Litteratur" in: Grundriß der romanischen Philologie, Hg. G. Gröber, 2. Bd. 2. Abt., Straßburg 1897, S. 447

16 Fr.Tr. Friedemann, "Der modernen Philologie wird fortdauernde Rücksicht auf die antike empfohlen" in: Archiv für das Studium der neueren Sprachen und Literaturen, Bd. 2, 1847 , S. $255-274$, hier S. 256

17 Ebda., S. 257

18 Ebda., S. 266 
Anspruch der Altphilologie auf die Neuphilologie übertragen. Die moderne Philologie ist also auch "Lehrerin der Jugend" ${ }^{19}$ und muß unterschiedliche Texte für die jeweilige Lebens- und Altersstufe finden, habe aber zugleich den wissenschaftlichen Ehrgeiz, ein Volk mit seinen charakteristischen Äußerungen zu betrachten.

Noch der "Vorschlag zu einer Enzyklopädie der modernen Philologie", den C. Sachs elf Jahre später in derselben Zeitschrift veröffentlichte, will die für die Altphilologie gültigen Grundbegriffe auf die moderne Philologie übertragen wissen. Auch Sachs umschreibt als Aufgabe der modernen Philologie, ein anschauliches Bild der modernen Völker zu geben, dabei aber zunächst die nach Literatur und Wissenschaft am meisten entwicklten $z u$ berücksichtigen ${ }^{20}$. Auch hier wird also ein Kanon besonders wertvoller Texte angestrebt. Dabei geht der Autor sogar so weit, das Spanische, das "zwar besonders in den norddeutschen Städten vielfach im praktischen Verkehr angewandt werde", aus der Neuphilologie zu eliminieren. Denn man dürfe nicht "den zufälligen Gebrauch für merkantile Zwecke mit dem inneren Werte und der welthistorischen Geltung" verwechseln ${ }^{21}$. Dem Postulat erzieherischen Wertes und dem Maßstab mustergültig entwickelten Volkscharakters entsprachen die volkstümlichen Texte, die seit der Romantik zum Kanon hispanistischer Beschäftigung wurden, offenbar nicht ausreichend - ein Fehlurteil, das bei Sachs zur Ablehnung des gesamten Fachs führt. In einem 1862 vor breitem Publikum an der Universität Kiel gehaltenen Vortrag über die Geschichte und Aufgabe der Philologie betont Georg Curtius seinerseits die hervorragende Bedeutung der griechisch-römischen Kultur und ihrer Philologie, die durch ihre Wiederentdeckung im 15. Jahrhundert wichtigste Grundlage der gesamten neueren Bildung wurde. Dies ist ihm aber nicht ein Grund, die Wiederentdeckung der Antike zum Gegenstand der Neuphilologie zu machen, sondern ein erneuter Beweis für die Notwendigkeit der vorausgehenden Beschäftigung des Neuphilologen mit der Altphilologie. Eine "Querelle des Anciens et des Modernes" deutet sich auf der Ebene der Philologien an, wenn Curtius die These ablehnt, es hätten nur Griechen und Römer Großes vollbracht. Er betont: "Jedes Volk entfaltet sein eigenes Wesen, das der Erforschung und Aufbewahrung Werth ist." 22 So hätten alle Philologien ein Ziel und eine Methode und wollten nichts anderes als den Zusammenhang der menschlichen Kultur zu erhalten. Die Kanonisierung unter den Nationalliteraturen, wie sie Sachs vornahm, ist damit überwunden. Curtius überträgt sogar auf die Neuphilologie jene Definition der Altphilologie, die nach A. Boeckh die Erkenntnis der geschichtlichen Entwicklung der Menschheit und nach Wolf als "Altertumswissenschaft" die gesamte Kultur mit allen Institutionen zu erhellen habe.

Gröber kritisiert 1888 diesen Ansatz, der in Anlehnung an die Methode der Altphilologie das geistige Leben der Romanen in seinem ganzen Umfang erfassen will. Er

1.9 Ebda., S. 270

20 C. Sachs, "Vorschlag zu einer Encyclopädie der modernen Philologie" in: Archiv für das Studium der neueren Sprachen und Literaturen, 13. Jg., 23. Bd., 1858, S. 1-8, hier S. 5

21 Ebda., S. 5 (Fußßnote)

22 Georg Curtius, Über die Geschichte und Aufgabe der Philologie, Kiel 1862, S. 25 f.; er zitiert den Oxforder Orientalisten Max Müller: Ziel der Philologie sei es "zu lernen, was der Mensch ist, indem sie lernt, was er gewesen ist." Ebda., S. 31 
betont damit, wie wenig das Konzept der klassischen Philologie auf die Neuphilologie anwendbar ist. Aufgabe letzterer sei nämlich, "die Erscheinungen des menschlichen Geistes in der Sprache, seine Leistungen in der künstlerisch behandelten Rede" ${ }^{23} \mathrm{zu}$ untersuchen. Damit werden Staaten-, Kultur-, Kunst- und Wissenschaftsgeschichte zu bloßen Grenzwissenschaften und die klassische Philologie wird zur Hilfswissenschaft.

Obwohl sich also die romanische Philologie "auf Schritt und Tritt an die lateinische Sprache und Literatur und nicht minder an griechisches Schrifttum gewiesen sieht ${ }^{24}$, könne sie eine Selbständigkeit gegenüber der klassischen Philologie beanspruchen. Natürlich kann diese Selbständigkeit am wenigsten in Grenzbereichen dokumentiert werden, die - wie z.B. die Auseinandersetzung mit den humanistischen Schriftstellern Spaniens - wohl auch deshalb eher vermieden werden.

Dennoch fehlen die Namen der Humanisten in der Wissenschaftsgeschichte der Romanistik dort nicht, wo sie ihr ein höheres Prestige gegenüber der Altphilologie verleihen können. Gröber nennt Humanisten wie Stephanus, Scaliger und G. Vossius, die sich gleichzeitig mit den lateinischen und den romanischen Sprachen beschäftigt haben. Von gesteigertem Selbstbewußtsein zeugt, daß Gröber diese Tatsache als Beleg dafür bewertet, daß sich sogar die klassische Philologie der Humanisten für Gegenstände der romanischen interessiert und damit die romanische Philologie bereits in der Renaissance zu ebenbürtiger Höhe erhoben habe ${ }^{25}$. So erscheinen denn auch als erste Bearbeiter der spanischen Sprache die ersten spanischen Humanisten, wie der Philologe Nebrija, oder der Reformator Juan de Valdés, der schon 1536 wie nach ihm 1606 Aldrete, den Ursprung der spanischen Sprache erforschte. Man sieht also diese Humanisten gleichzeitig als klassische Philologen und als Hispanisten.

\section{Gattungen}

Im Konkurrenzkampf der Philologien treten die Humanisten daher nicht als Vertreter der Dichtung in Erscheinung. Da Romanzenliteratur und Drama im Vordergrund des Interesses standen, fand humanistische Prosa kaum Beachtung, zunal da ihr eher rhetorischer als poetischer Charakter zugeschrieben wird. Im folgenden sei daher die Beurteilung des humanistischen Schrifttums als Texttyp vorgeführt.

Bouterwek hebt 1804 in seinen Ausführungen zu Pérez de Oliva hervor, daß gerade in der ersten Hälfte des 16. Jahrhunderts von den spanischen Gelehrten auf eine "Scheidung der Poesie von der Beredsamkeit" 26 sehr viel Wert gelegt wurde. Pérez de Oliva, der mit seiner Schrift über die Menschenwürde ein humanistischer Autor ist, wird also den gelehrten Autoren zugerechnet, die nach Bouterwek viel zu

23 G. Gröber, "Aufgabe und Gliederung der romanischen Philologie" in: Grundriß der romanischen Philologie, Hg. G. Gröber, Bd. 1, Straßburg 1888, S. 140-154, hier S. 146

24 Ebda., S. 149

25 G. Gröber, "Geschichte der romanischen Philologie" in: Grundriß der romanischen Philologie, Bd. 1, a.a.O., S. 1-139, hier S.11

26 Friedrich Bouterwek, Geschichte der spanischen Poesie und Beredsamkeit, Göttingen 1804 (Geschichte der Künste und Wissenschaften, 3. Abt., 3. Bd.), S. 303 
wenig bekannt seien. Es lohne sich der Mühe, schreibt er, "genauere Anleitung zur Kenntnis einiger guter spanischer Prosaiker zu geben, deren Namen in der allgemeinen Geschichte der neueren Beredsamkeit kaum einmal genannt sind." 27 Bouterweks Aufforderung sollte noch lange ungehört bleiben.

Denn die Poesie war es, die in erster Linie - auch Bouterwek - interessierte. Er sah die spanische Literatur, die die nationale Identität verkörpere, im Schauspiel. So wählte auch Adolf $F$. von Schack für seine Literaturgeschichte die dramatische Literatur Spaniens nicht ohne Grund. Wie Schlegel ist auch er der Meinung, daß das Volkstümliche gerade in dieser Gattung sichtbar wird ${ }^{28}$. Auch in der Literaturgeschichte des Amerikaners Ticknor, der von 1815 bis 1817 in Göttingen studiert hatte, hat die humanistische, an der Antike orientierte Literatur in den Hintergrund zu treten. Dieses Werk lag 1852, bereits drei Jahre nach seinem Erscheinen, in der von Nikolaus H. Julius bearbeiteten und ergänzten deutschen Übersetzung vor und wurde von Prescott mit der Bemerkung angekündigt, "daß die Spanier, deren Sprache, ebensosehr wie die der Franzosen und Italiener, auf lateinischer Unterlage ruhte, sich dennoch enthielten, ihre Literatur gleich jenen Völkern auf die classischen Muster des Alterthums zu gründen, und es vorzogen, sich mehr dem romantischen Geiste der nördlichen germanischen Völker anzuschließen." ${ }^{29}$ Dies führt Prescott auf den vorherrschenden Einfluß der gotischen Grundbestandteile ihres Charakters zurück. Ein prinzipiell konstatiertes Fehlen von imitatio und aemulatio als Prämisse ist also ein weiterer Erklärungsgrund für die Geringschätzung humanistischer Prosa.

Dennoch wird bei Ticknor auf einige spanische Humanisten eingegangen. Jedoch werden sie als bloße Nachahmer der Italiener, die ihrerseits die antiken Autoren Cicero und Seneca nachgeahmt hätten, also nicht als unmittelbare, sondern mittelbare Nachahmer abgewertet. Erörtert werden z.B. Werke von F. de Villalobos, Pérez de Oliva, Morales, Cervantes de Salazar, A. de Guevara, Juan de Valdés, A. de Nebrija, Las Casas, Sepúlveda und Pedro Mexía. Die beliebte "Silva" des letzteren ahme zwar die römischen und italienischen Meister nach und habe dem Autor mehr Ruhm als irgendeinem anderen spanischen Prosaschriftsteller seiner Zeit verschafft, bleibe jedoch in gedanklicher Höhe weit hinter den Vorbildern zurück ${ }^{30}$.

Trotz der genannten Autoren konstatiert Ticknor einen Mangel an guter lehrender Prosa im 16. Jahrhundert. Er erklärt ihn mit dem Hinweis auf mögliche Gefahren:

\section{$2 \tau$ Ebda., S. $303 \mathrm{f}$}

28 Adolph Friedrich von Schack, Geschichte der dramatischen Literatur und Kunst in Spanien, Bd. 1, Berlin 1845; Schack will zeigen, "wie das wahrhaft Große und Originale in der Poesie nur auf dem Boden der Volksthümlichkeit gedeihen könne, wie namentlich das Drama in Geist und Form duch die Geschichte und den Charakter des jedesmaligen Volkes auf's strengste bedingt sei, und wie es, um zum ächten Nationalschauspiel zu erblühen, aus dem innersten Kerne einer Nation und im Zusammenhange mit ihren volkspoetischen Sagenkreisen und ihrer Geschichte erwachsen müsse." Ebda., S. XVII f

29 Georg Ticknor, Geschichte der schönen Literatur in Spanien, dt. mit Zusätzen von N.H. Julius, 1. Bd., Leipzig 1852, S. XIV

30 "Seine Erfindungsgabe reicht niemals aus, seinen Gegenstand auf neue und kräftige Weise zu betrachten." Ebda., S. 419 
"Fast alle Schriftsteller verfielen, weil man sie daran hinderte, sich der Logik einer weisen und freisinnigen Philosophie zu bedienen, in Gelehrttuerei, indem sie, wo es nur immer möglich war, sich gern auf Gewährsmänner stützten." ${ }^{31}$ Daher sei diese Prosa von zahlreichen Zitaten aus den Kirchenvätern, den scholastischen Philosophen und der Bibel durchsetzt. Aus diesem Druck erkläre es sich.auch, daß die belehrende Prosa steif und übergelehrt wurde und sich dahingeschleppt habe, während "die minder verdächtigen und unterdrückten Teile spanischer Literatur, die dramatische und lyrische Dichtung, am höchsten standen." 32

Vor dem Hintergrund von Ticknors in Deutschland vielbeachteter Literaturgeschichte wird verständlich, daß auch in der Auswahl von Musterstücken aus den Werken spanischer Prosaisten von L. Lemcke 1855 die Humanisten Guevara und L. de León bewußt ausgelassen werden. Lemcke rechtfertigt dies in der Vorrede. Ihm scheine A. de Guevara "ungeachtet der Vorzüge seiner Schreibart, nicht bedeutend genug, um in einem Handbuche einen besonderen Platz einzunehmen" ${ }^{33}$. Er übergeht auch L. de León, "weil seine prosaischen Schriften bei allem stilistischen Wert doch nur eine untergeordnete Wichtigkeit haben." 34 Anders jedoch als Ticknor verurteilt er die humanistische Literatur nicht pauschal. Denn den "Diálogo de la dignidad del hombre" zählt er zu den wichtigen Werken der spanischen Literatur. Von ihm als dem ältesten "wirklich klassischen Muster didaktischer Prosa" ${ }^{35}$ druckt er Auszüge, wie auch von Texten aus Cervantes de Salazar.

Auch 1867 in der Anthologie und Literaturgeschichte von H. Dohm wird Pérez de Olivas Dialog als "Abhandlung, die durch ihren gediegenen Inhalt und ihre classische Sprache zu den besten spanischen Werken didaktischer Prosa gehört" ${ }^{36}$ gewürdigt. Er fehlt auch nicht in der Schrift von A. Schmidt mit dem Titel "Was muß man von der spanischen Literatur wissen?" von 1900, in der zahlreiche Autoren der didaktischen Prosa des 16. Jahrhunderts aufgeführt werden. Uneingeschränkt positiv werden sie jedoch nicht bewertet. Denn es gebe kaum "einen Gegenstand, kaum ein Gebiet, über welches nicht solche, oft recht langweilige Abhandlungen geschrieben worden wären." ${ }^{37}$ Als "Abhandlungen" bezeichnet, werden die humanistischen Schriften aus dem Bereich der "schönen Literatur" eliminiert. 1903 wählt R. Beer für sie sogar die

31 Georg Ticknor, a.a.O., Bd. 2, S. 314

32 Ebda., S. 315

33 Ludwig Lemcke, Handbuch der spanischen Litteratur. Auswahl von Musterstücken aus den Werken der klassischen Spanischen Prosaisten und Dichter von den ältesten Zeiten bis auf die Gegenwart, mit biographisch-litterarischen Einleitungen, Bd. 1, Leipzig 1855, S. VIII

34 Ebda.

35 Ebda., S. 180

36 H. Dohm, Die spanische National-Literatur in ihrer geschichtlichen Entwicklung. Nebst den Lebens- und Charakterbildern ihrer classischen Schriftsteller und ausgewählten Proben aus den Werken derselben in deutscher Uebertragung, Berlin 1867, S. 237

37 Albert Schmidt, Was muß man von der spanischen Litteratur wissen? Gemeinverständlich beantwortet, Berlin 1900, S. 103 
Gattungsbezeichnung "Fachliteratur" 38 .

Schließlich ordnet P.A. Becker 1904 Pérez de Oliva, A. de Guevara, J. de Valdés und P. Mexía den spanischen Moralisten zu. Deren "Moralliteratur" sei "ein spezifisches Erzeugnis des humanistischen Zeitalters und vielleicht der entsprechendste Ausdruck seines Geistes." ${ }^{39}$ Gerade in Spanien, wo die Kirche mißtrauisch jede Äußerung selbständigen Denkens betrachte, und Autoren wie Servet und Vives so gut wie Ausländer seien, sei diese Moralliteratur "das eigentliche Gebiet, auf dem die Kunstform der Prosa ihre bewußte Ausarbeitung und Vervollkommnung fand." 40

\section{Einfluß der Kirche}

Auf den Einfluß der Kirche, der eine freie Entfaltung des Humanismus verhindert habe, weisen viele Autoren hin. Er wurde allerdings nicht nur als Last gesehen. Nach Clarus z.B. hat die Kirche bereits im Mittelalter die klassischen Studien gepflegt und damit erst den Boden für die Renaissance geschaffen. Lange vor Klemperer hatte Clarus 1846 betont, daß sich gegen Ende des 15. Jahrhunderts, während sich im übrigen Europa das Christentum längst konsolidiert hatte und unter Verfallserscheinungen litt, die spanischen Zustände trotz aller politischen Zerrissenheit "eine sichere christliche und kirchliche Positivität" ${ }^{11}$ darstellten. Dort, wo allerdings das klassische Altertum in Spanien Aufnahme fand, war dies nach Clarus nur möglich, weil das Mittelalter die klassische Kultur tradiert hatte ${ }^{42}$. Wäre man wirklich im Mittelalter in Dunkelheit und Barbarei versunken, dann hätte man kaum diese Kulturschätze willkommen heißen können.

Clarus widerspricht der These, die Reformatoren hätten mit den Humanisten und mit der Rückbesinnung auf die literarischen Schätze des Altertums ein Zeitalter der Dummheit und Finsternis beendet. Dagegen hält er, daß sie sich der Antike nur aus taktischen Gründen bedient haben. Dies zeige Luther, als er mit den Humanisten brach: "Sobald er durch andere Mittel seine Partei [...] stark wußte, fiel er vom Humanismus geradezu ab und erblickte in der classischen Gelehrsamkeit nichts mehr als ein glänzendes Laster." 43

Da nämlich die Schätze des Altertums durch die Kirche tradiert seien, sei "die Alterthumswissenschaft, eine durchaus katholische Disziplin, in der Kirche geboren,

38 Rudolf Beer, Spanische Literaturgeschichte, Bd. 2, Leipzig 1903, S. 89

39 Philipp August Becker, Geschichte der Spanischen Literatur, Straßburg 1904, S. 44

40 Ebda.

41 Ludwig Clarus, Darstellung der spanischen Literatur im Mittelalter, Mainz 1846, Bd.2, S. 527; dieser christliche Geist, den Spanien in die schöne Literatur einführte, "widerstand der heidnischen Werbung und machte es sich zur Aufgabe, die göttliche Wahrheit und Ordnung anzudeuten und zu veranschaulichen in seinen literarischen Bildern und Gestaltungen." Ebda., S. 528

42 "Schon die begeisterte Annahme, welche die aus den eröffneten Fundgruben des classischen Alterthumes geförderte Ausbeute so allgemein fand, setzt eine schon ansehnlich vorgeschrittene Geistesbildung voraus." Ebda., S. 534

43 Ebda., S. 535 
aufgesäugt und immer aufs Großartigste unterstützt." 44 Wenn Clarus selbst durch ausführliche Erörterung der mittelalterlichen didaktischen Literatur belegt, wie intensiv man bereits im Mittelalter um das Studium der Antike bemüht war, dann erscheint der Humanismus nicht als Renaissance, sondern als eine unter vielen Etappen, die sich kontinuierlich aneiander anschließen.

Kontinuität seit dem Mittelalter sieht der Oberlehrer Paul Förster in der Aufgabe der Inquisition, über deren Bedeutung er 1890 eine Abhandlung im Jahresbericht der königlichen Realschule zu Berlin veröffentlicht. Trotz inquisitorischer Aktivität habe es jedoch auch in der ersten Hälfte des 16. Jahrhunderts nicht an scharfer Kritik der Geistlichkeit gemangelt. Für Förster ist diese Kritik nichts anderes als die Kehrseite von inniger Frömmigkeit, bei der auch "der Humanismus in Spanien gute Aufnahme finden" ${ }^{45}$ konnte. "Aber er zeigt sich uns hier nicht in seiner heiteren, spielenden, rein verstandesmäßigen Art; er stellt den Geist in den Dienst sittlicher, reformatorischer Gedanken." ${ }^{46}$ Da sich der Spanier nicht auf den Standpunkt des ironisch lächelnden Humanisten zurückziehen könne, finde man "in Spanien im Ausgange des 15. Jahrhunderts parallel der deutschen eine spanische Reformation." ${ }^{47}$ Deren Ansätze der Erasmusrezeption bei Vives oder Valdés seien von der Inquisition bis 1570 vollständig niedergeworfen worden. Damit habe Spanien nicht nur theologische Ketzer, sondern auch ausgezeichnete Schriftsteller verloren ${ }^{48}$. Der Verfall der Wissenschaften und guten Sitten seien die Folge gewesen. Die Bedeutung der Reformatoren aber, darauf weist Förster hin, sei seit kurzem durch die Arbeiten von Wiffen und Boehmer gut übersehbar.

Der spanische Humanismus zeigt sich also immer religiös, sei es, daß er wie bei Vives im Einklang mit der offiziellen Theologie erscheint, sei es, daß er wie bei Valdés den Weg zur Reformation und zur Heterodoxie einschlägt. Diese reformatorische Dimension der spanischen Humanisten war es, die das Interesse des Engländers Wiffen und des Straßburger Theologen und Romanisten Boehmer hervorgerufen hat. Boehmer setzte Wiffens Werk fort und veröffentlichte ab 1874 Biographien und Schriften der spanischen Reformer zur Zeit von Erasmus unter Benutzung der Wiffenschen Manuskripte ${ }^{49}$. Er konnte dabei auf die "Historia de los protestantes españoles" von Adolfo de Castro von 1851 und die "Historia de los heterodoxos españoles" (1877-82) von M. Menéndez Pelayo zurückgreifen. Die Bedeutung des Erasmus in Spanien hatte Boehmer bereits 1862 in einem Artikel über spanische Übersetzungen des Erasmus lange vor Bataillon - hervorgehoben ${ }^{50}$. Spanische Schriftsteller wie Valdés interessier-

44 Ebda.

45 Paul Förster, "Der Einfluß der Inquisition auf das geistige Leben und die Litteratur der Spanier" in: Jahresbericht der Königlichen Realschule zu Berlin. Realgymnasium, Berlin 1890, S. 11

46 Ebda.

47 Ebda., S. 13

48 Ebda., S. 22

49 Edward Boehmer, Spanish Reformers of two centuries from 1520. Their Lives and Writings, Straßburg/London, 1. Bd. 1874 (Bibliotheca Wiffeniana)

50 Edward Boehmer, "Erasmus in Spanien" in: Jahrbuch für romanische und englische 
ten also nicht, insofern sie als Humanisten ein neues Verhältnis zur Antike gewannen, sondern insofern sie Reformideen in die christliche Kirche brachten. Auch bei diesem theologischen Ansatz wird weitgehend die Betrachtung der humanistischen Auseinandersetzung der Würdigung einer neuen christlichen Religiosität geopfert.

Abschließend und zusammenfassend läßt sich festhalten, daß Klemperers These Gedankenelemente aufgriff, die sich bereits bei Herder und Schlegel fanden. Dazu gehören die Betonung des arabischen Einflusses auf mittelalterliche Literatur und Ritterkult, ebenso wie die Suche nach jener Dichtung, die Ausdruck des Volkscharakters ist. Es hat sich nun gezeigt, daß die romantische Perspektive die Auswahl der spanischen Literatur im 19. Jahrhundert so beeinflußt hat, daß der Blick für die gelehrte humanistische Literatur, die im Falle Spaniens ja nur eine fremde, römischitalienische Literatur erschlossen hätte, verlorenging. Hinzu kam, daß für die neuphilologische Hispanistik, die sich gegenüber der Altphilologie profilieren wollte, die Beschäftigung mit den spanischen Humanisten kein geeignetes Feld darstellt, da der Humanismus als Kapitel der Altphilologie verstanden wurde, und sich kaum eignete, die Selbständigkeit der Neu- gegenüber der Altphilologie festzuschreiben. Deren Methode der Kanonbildung übernahmen zunächst auch die Neuphilologien nicht nur für ihre eigenen Literaturen, sondern auch für ihren Konkurrenzkampf untereinander. Hier wurden die Humanisten vor allem dort zitiert, wo sie das hohe Niveau der Neuphilologie bereits in der Renaissance dokumentierten. In der spanischen Dichtung selbst jedoch erscheint die imitatio der Antike weder charakteristisch, noch wesentlich. Die Texte der Humanisten, die der Rhetorik und nicht der Poesie zugeordnet wurden, wirkten wenig originell und zudem noch mit Rücksicht auf die Inquisition mit Zitaten überladen. Man grenzte sie aus dem eigentlich Literarischen aus und ordnete sie der Fach-oder Moralliteratur zu.

Die Dominanz der Kirche erschien nicht unbedingt als Hindernis für humanistische Studien. Im Gegenteil, sie hatte nach Clarus seit dem Mittelalter die Altertumswissenschaft als katholische Disziplin tradiert. Der Humanismus erscheint so als Kontinuität und nicht als Renaissance. Wenn nun auch Reformation und Humanismus als weitgehend voneiander getrennte Strömungen gelten, dann war es dem theologisch interessierten Böhmer möglich, zahlreiche Humanisten in theologischer Perspektive als Reformatoren vorzustellen, ohne deren humanistische Arbeit zu würdigen.

Diese Gedenkengänge, die Klemperer wohl kannte, bilden natürlich weder ein kohärentes Ganzes, noch eine kontinuierliche Entwicklung. Obwohl mit unterschiedlichen Intentionen von unterschiedlichen Positionen ausgehend, zeichnen sie dennoch einen Horizont $a b$, aus dem erklärbar wird, warum eine unvoreingenommene und vorurteilsfreie Betrachtung der spanischen Humanisten nicht möglich war.

Literatur, Bd. 4, 1862, S. 158-165 